\title{
A Modular Robotic System Using Magnetic Force Effectors
}

\author{
Brian T. Kirby, Burak Aksak, Jason D. Campbell, James F. Hoburg, \\ Todd C. Mowry, Padmanabhan Pillai, Seth Copen Goldstein
}

\begin{abstract}
One of the primary impediments to building ensembles of modular robots is the complexity and number of mechanical mechanisms used to construct the individual modules. As part of the Claytronics project-which aims to build very large ensembles of modular robots-we investigate how to simplify each module by eliminating moving parts and reducing the number of mechanical mechanisms on each robot by using force-at-a-distance actuators. Additionally, we are also investigating the feasibility of using these unary actuators to improve docking performance, implement intermodule adhesion, power transfer, communication, and sensing.

In this paper we describe our most recent results in the magnetic domain, including our first design sufficiently robust to operate reliably in groups greater than two modules. Our work should be seen as an extension of systems such as Fracta [9], and a contrasting line of inquiry to several other researchers' prior efforts that have used magnetic latching to attach modules to one another but relied upon a powered hinge [10] or telescoping mechanism [12] within each module to facilitate self-reconfiguration.
\end{abstract}

\section{INTRODUCTION}

Advances in manufacturing and electronics open up new possibilities for designing modular robotic systems. As the robots become smaller, it becomes possible to use force-at-adistance actuators-e.g., actuators which cause one module to move relative to another via magnetic or electric fields external to the modules themselves. Furthermore, as the cost and power consumption of electronics continue to decrease, it becomes increasingly attractive to use complex electronics rather than complex mechanical systems. In this paper, we explore how a single device that exploits magnetic forces can be harnessed to unify actuation, adhesion, power transfer, communication, and sensing. By combining a single coil with the appropriate electronics we can simplify the robotreducing both its weight and size-while increasing its capabilities.

The robots described in this paper are the result of our explorations into the underlying ideas of the Claytronics project [4], which is investigating how to design, build, program, and use ensembles comprised of massive numbers of robotic modules. Thus, one of the main driving design

This work was supported in part by DARPA/SPAWAR N66001-04-189XX, NSF CNS-0428738, and Intel Corporation. We want to thank the members of the claytronics group for their many valuable insights.

Kirby, Goldstein in SCS at CMU, 5000 Forbes Ave, 15213

$\{$ bkirby, seth $\}$ acs. cmu.edu

Aksak in ME at CMU aksakame. cmu. edu

Hoburg in ECE at CMU hoburg@ece. cmu . edu

Campbell, Mowry, Pillai at Intel Pittsburgh Research

\{jason.d.campbell, todd.mowry,

padmanabhan.s.pillai\} @intel.com

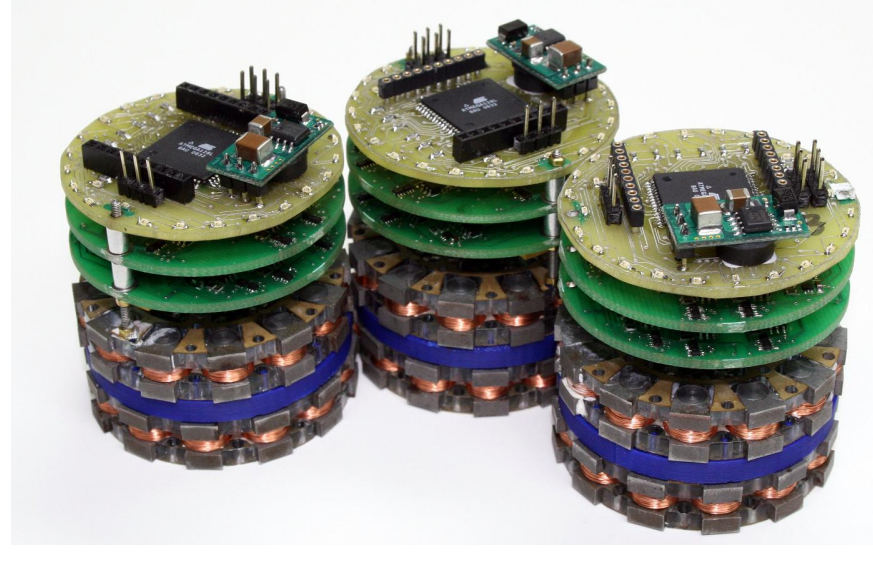

Fig. 1. Three magnetic $45 \mathrm{~mm}$ planar catoms. Videos demonstrating their movement capabilities are available at http://Www.cs.cmu.edu/rclaytronics/iros07/planarcatom/.

criteria for any individual mechanism is: will it support scaling the ensemble to larger numbers of units?. A direct outgrowth of this design criteria is that each unit in the ensemble must be inexpensive, robust, and easy to manufacture. Hence mechanisms used for locomotion, adhesion, communication, etc., must be as simple as possible. One way to achieve this is to use inexpensive and robust resourcese.g., computation-to reduce mechanical complexity. Furthermore, since we are interested in the ensemble as a whole, we do not require that individual units be self-sufficient. As long as individual units can contribute to the overall motion of the ensemble, they do not need the ability to move independently within the greater environment. We call this design principle the ensemble axiom: each unit contains only the minimum abilities necessary to contribute to the aggregate functionality of the ensemble.

Choosing the right mechanism for locomotion is a key design decision. In addition to scalability, the size of the unit must also be taken into account. At the macroscale, complex mechanisms such as motors are effective. However, as units scale down in size other approaches become viable, taking advantage of increasing surface-to-volume ratio and decreasing of inertial moments. Our current robots, which we call planar catoms $^{1}$, are small enough that we can explore a mechanism designed around magnetic field forceat-a-distance actuators. As the units decrease further in size, actuators based upon electric field forces become viable and are appealing because they use less current, produce less

\footnotetext{
1 "Catom" is short for "claytronics atom."
} 
heat, and weigh less than magnetic actuators. Even smaller units could harness surface forces such as surface tension or Van der Waals' forces. The size scale also affects power transfer and storage: because electrical resistance increases as contact size decreases, direct electrical connections between robots become increasingly impractical. We chose the centimeter scale for our initial prototypes to keep the smallscale prototyping costs of our onboard circuitry reasonable.

In keeping with our design principle, we demonstrate $45 \mathrm{~mm}$ diameter cylindrical modular robots (see Figure 1) that can move in a plane and use a single, no-movingparts mechanism-an electromagnetic coil-for locomotion and adhesion (Section III), power transfer (Section IV), and communication and neighbor sensing (Section V). The ability to implement a number of features using the same mechanism allows us to reduce the weight, volume, and overall complexity of the unit.

\section{RELATED WORK}

The effort to produce reliable and robust modular robotic systems has led researchers to explore a large design space of mechanisms for locomotion, adhesion, communication, and power. Ostergaard, et al. survey different locomotion and adhesion mechanisms for self-actuating robots in [5].

Of the many research efforts the most relevant to our work is Fracta [9]. Fracta is a two dimensional modular robot which uses a combination of permanent magnets and electromagnets for locomotion and adhesion. It is the only other internally actuated system which has no moving parts. As in our planar catoms, to move a module requires communication between the moving module and its neighbors. The two main differences between Fracta and planar catoms are due to changes in underlying technology and the use of permanent magnets. Fracta modules are constrained to be in a hex-lattice whereas the planar catoms have additional actuators and can be arranged in a cubic or hex lattice, as well as more arbitrary formations. Significant advances in VLSI enable us to create smaller, lighter units which do not use permanent magnets. We also harness the magnets for more than locomotion and adhesion, i.e., the magnets also serve as the main mechanism for power transfer, sensing, and communications.

Planar catoms are our first step along the path towards realizing three dimensional claytronics. Part of their raison d'etre is to understand the ensemble axiom and how the tradeoff between individual unit hardware complexity and computation affects design. As such, work in externally actuated modular robots is also relevant. For example, neither programmable parts [2] nor 3D stochastic robots [11] have any moving parts. Both of these systems simplify each robot by using external forces for actuation. The robots rely on the external forces and move stochastically, adhering to each other under control of the program running on the robot. The ensemble principle is carried even further in the latter project; robots are unpowered until they adhere to a powered robot.
Earlier prototypes of the planar catoms described in this paper have been demonstrated at AAAI [6] and have been briefly described in the general media. This paper is the first complete description and introduces the ideas behind using a single device (electromagnets) to implement locomotion, adhesion, power transfer, communication, and sensing.

\section{LOCOMOTION}

Using the ensemble axiom as a guiding design principle requires that we design very small robotic modules capable of actuating relative to one another. As discussed earlier, to make reliable modules that can be readily scaled down in size, we have taken the extreme position of eliminating all moving parts within our robotic modules. Motion without moving parts is achieved instead by the use of force-at-adistance actuation between modules. The mechanisms that work well for this purpose are highly dependent on the absolute scale of the module design. We chose the centimeterrange for our prototypes, as it was the smallest size we could implement self-contained modules using commercially available electronic components and circuit board design techniques. At this scale we are well beyond the practical application of surface tension, Van der Waals force, or electrostatic attraction, and therefore employ electromagnetism for our actuation.

\section{A. Relative Motion using Pairs of Electromagnets}

In keeping with the ensemble axiom, planar catom motion requires two modules to perform the simplest locomotion. Our actuation methodology can be likened to a rotary linear motor, e.g. a stepper motor [3] in which the stator and rotor are mechanically decoupled into two separate, identical modules set side by side. Rather than permanent magnets, both catoms generate their fields with the appropriate polarities via electromagnets. Catoms in contact may orbit each other in a clockwork fashion by simultaneously activating electromagnets adjacent to the pair currently in contact. The magnetic force will create a torque that pivots the two catoms about the edge and onto the next face. Once in position, the catoms can again activate the next adjacent pair and continue their orbit.

In ideal conditions, this motion takes as little as $50 \mathrm{~ms}$ to complete one step, or $1.2 \mathrm{~s}$ for a complete revolution. However, unlike a stepper motor, which is carefully designed with tight mechanical tolerances and excellent axial alignment, our catoms must regularly deal with mechanical misalignment both in and out of the plane of motion. As magnetic force diminishes proportional to the cube of the distance, these small misalignments seriously compromise the efficiency of our motion. When using simple open-loop control, it is necessary to power the coil for much longer than needed for the ideal case, to give the catoms time to exert themselves over farther distances. In previous generations of prototypes, this conservative on-time has been 10-20x longer than the ideal. This variability in performance thus has a large effect on power efficiency, and suggests why closed- 


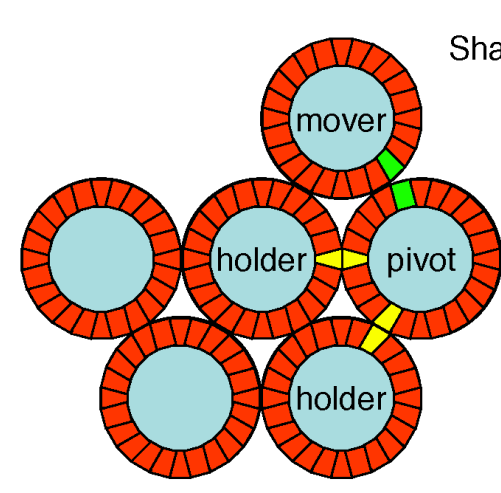

(a)

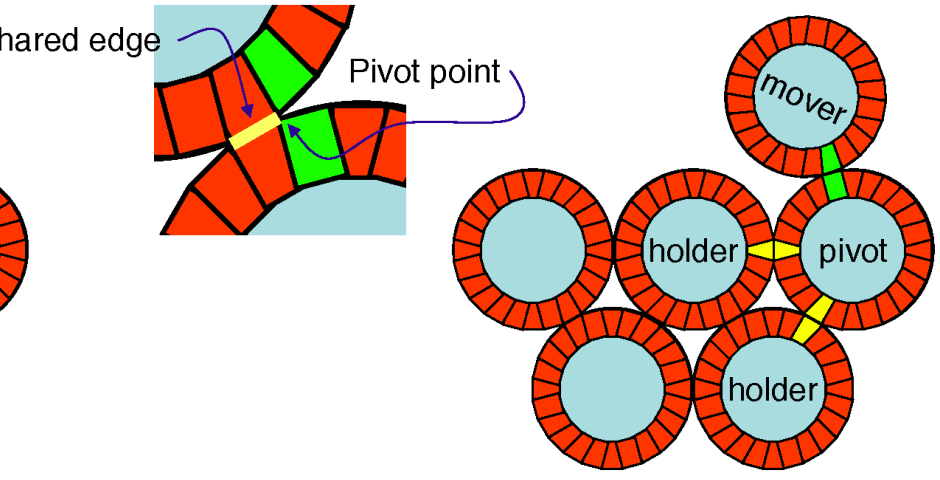

(b) (c)

Fig. 2. A typical movement scenario. (a) is the start configuration. (b) is a blow up of the mover-pivot pair. (c) is the final configuration. The yellow magnets exert a small holding force. The green magnets exert a large force to move the mover around the pivot.

loop control is highly desirable in our system yet generally not implemented in standard stepper motors.

\section{B. Ensemble Motion}

While the basic motion primitive requires the participation of only two catoms, any motion which performs actual work, i.e., motion which changes the configuration of the ensemble, requires the involvement of more than two catoms. We distinguish three types of catoms in ensemble motion: movers, pivots, and holders. The mover catom moves around a pivot catom with respect to the rest of the ensemble. The other modules surrounding the pivot catom are holders, and keep the pivot catom in formation as the mover moves around it.

In a basic movement scenario, the pivot catom and all its neighbors except the mover catom actuate their magnets with a low holding force (the yellow magnets in Figure 2a). The mover and pivot then energize the magnets used to move the mover catom (the green magnets in Figure $2 \mathrm{a}$ and $\mathrm{b}$ ). This causes the mover catom to pivot around the edge it shares with the pivot catom, resulting in Figure 2c.

\section{Magnet Design and Constraints}

Our initial investigations focused on permanent magnet solutions, as these provide a holding force without a static power dissipation. We experimented with programmable magnetics, using AlNiCo magnets that can be made to change polarity when subjected to brief pulses from an encompassing electromagnet. Unfortunately these were too weak to generate useful forces for our application. We also considered using the surrounding electromagnet as our primary actuator, using the soft magnetic material only as a passive holding actuator, but the AlNiCo has poor permeability and low saturation, preventing the electromagnets from generating the requisite forces. By using a more traditional electromagnet core material, we were able to design magnets with effective force. Additionally, as we will see in later sections, the electromagnets can be used for other purposes. Thus, the planar catoms use the same electromagnets for

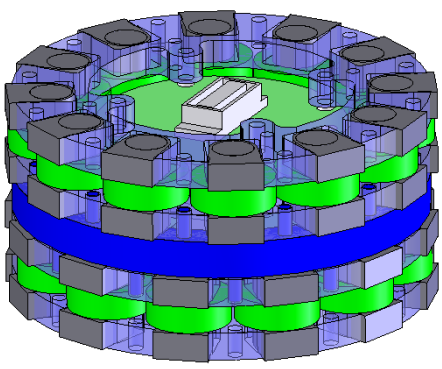

Fig. 3. The main body of the catom is comprised of two rings of magnets offset by 15 degrees.

locomotion, adhesion, power delivery, communication, and sensing.

The design constraints involved in determining the size, shape and number of magnets are numerous. First and foremost, the magnets must provide sufficient torque to rotate a catom around a shared edge (e.g., the highlighted edge in Figure $2 \mathrm{~b}$ ). The torque required is influenced by catom mass and diameter, as well as the friction between a catom and the floor. The electromagnets themselves are quite heavy as they have a large copper winding and both the core and flux shunt are composed of steel. The minimum amount of core material is dictated by magnetic flux saturation-reducing the crosssectional area of the core would dramatically reduce magnet strength. The copper coil is limited by the power densityreducing the cross-sectional area of the coil would force proportionally higher current through less material, increasing heat dissipation and dramatically lowering the effective duty cycle of the actuator. Friction cannot be lowered arbitrarily as low friction constants make the movement between catoms unstable (e.g., the catoms tend to fly away from each other).

In addition to being strong and compact, the magnets must also be carefully shaped so that they can be placed around the circumference of the catom without interfering with each other. Furthermore, we want to restrict the lattice packing as little as possible, supporting at least hex and cubic lattices. 


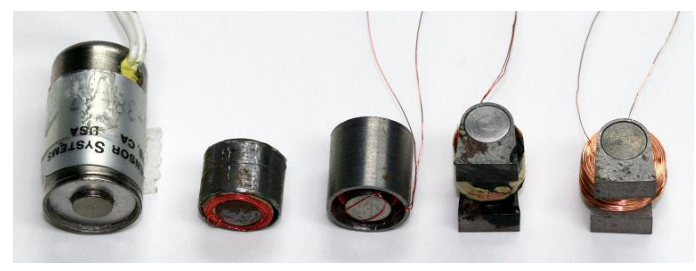

Fig. 4. A progression of catom magnet designs. The rightmost magnet is our current revision.

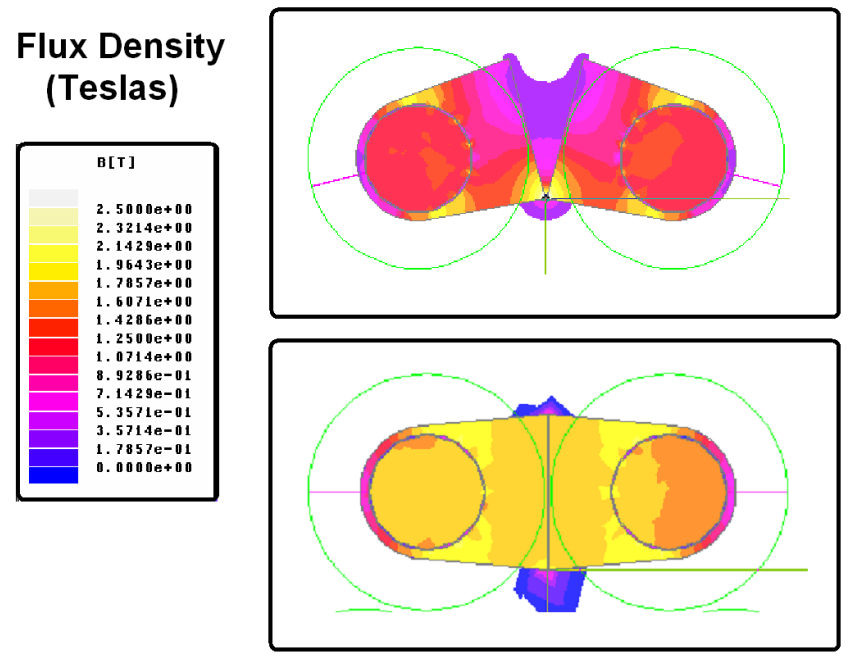

Fig. 5. At the start of a motion (top), the flux saturates the bottom tip of each magnet, generating the initial torque of $12 \mathrm{mN}-\mathrm{m}$. At the end (bottom), the flux is evenly distributed and provides far greater holding torque $(200 \mathrm{mN}$ m) for the same power.

We used these three factors and the fact that magnetic force diminishes with the cube of the distance to determine that 24 magnets would be the best balance of constraints. To prevent the magnet core material from being close enough to cause interference, we stagger the magnets in two offset rings of twelve spaced $4 \mathrm{~mm}$ apart as in Figure 3. This has the added benefit of giving us larger effective area for our coil windings. Using more than two rings is prohibitive, because it begins to introduce significant out-of-plane torques as the magnet layers become farther and farther from the friction plane. With each individual magnet designed to maximize flux density, reduce saturation, and minimize overheating, we finally consider resistance and wire gauge so that our voltage and current requirements can be met with high density surface mount components such as MOSFETs.

Commercially-available, off-the-shelf electromagnets proved insufficient for our actuators. They did not fit well in our cylindrical geometry and had far too conservative power usage and duty cycles to satisfy our torque needs. We chose to design our own magnets. After several iterations (shown in Figure 4), our current design places the coil vertically and uses two thick trapezoidal end plates that combine to form a horseshoe electromagnet. The ends of the horseshoe are flat to improve catom-to-catom alignment. The sharp edges of the end plates also provide a natural pivot point. (Initially we tried rounded ends, but this results in an unstable system.) The current design also helps ameliorate the loss of magnetic force due to the initial gap between modules, as at the start of a move operation, the actuating magnets already have a narrow but complete flux path. The flux paths may be seen in Figure 5 at both the initial and final stage of a motion step.

The resulting system has 24 magnets arranged in two offset rings of 12 magnets forming a faceted, self-aligning structure with a large potential excitation capability and acceptable duty cycles. The coil height is $3 \mathrm{~mm}$ and has 452 turns of 39 gauge wire around a $4.4 \mathrm{~mm}$ AISI1010 steel core and presents its flux at the catom's perimeter, $4.2 \mathrm{~mm}$ from the center of the coil, via two $3 \mathrm{~mm}$ thick flux shunts. When energized at full power at the start of motion, these coils are capable of co-generating a torque of $12 \mathrm{mN}-\mathrm{m}$. The worst-case torque needed, that of moving one catom about a second fixed catom, is given by the formula $\tau=m g r \mu$ and is around $3 \mathrm{mN}-\mathrm{m}$ given a $.105 \mathrm{~kg}$ module assuming a low friction coefficient of .12. When energized in a holding position, they can generate over $200 \mathrm{mN}-\mathrm{m}$ at full power. By using a small fraction of full power we can generate adequate holding torque without danger of overheating the coils.

\section{Control Circuits}

When moving a catom the magnets require high excitation currents for short periods of time. Conversely, when holding two catoms together, the magnets are next to each other and thus require very little excitation but should remain on continuously. The magnet control circuit is designed to support both situations. This greatly simplifies ensemble control, as without a holding force, accurate synchronization between many catoms would be required if they were to hold one catom in place while another rotated about it. We also need control of the polarity to coordinate an attractive force between two separate catoms. Consequently, our drivers must be capable of independent, bidirectional delivery of over 30 Watts in sub-second bursts, as well as delivering a few watts over multi-minute periods. Fortunately, modern MOSFETs support the required power densities in packages small enough to fit the drivers for the entire magnet array onto the catom itself.

Our initial controller design implemented 24 full bridges for completely independent control of each magnet. Fitting everything necessary into a $44 \mathrm{~mm}$ diameter printed circuit board was a laborious process and greatly increased manufacturing costs. As we continued to investigate the motion and lattice constraints, we realized that no movement circumstances would ever require us to activate more than one of any four consecutive electromagnets around the 24gon. By separating the full bridges into half-bridges, and using one shared half bridge between these four, we were able to reduce the number of half bridges from 48 to 30 , as well as multiplex the magnet control signals. This dramatically reduced the circuit density, as shown in Figure 6, and made pulse width modulation (PWM) signal generation practical for our control signals. PWM allows for simple open-loop current control. Thus, in addition to a full-duty, 


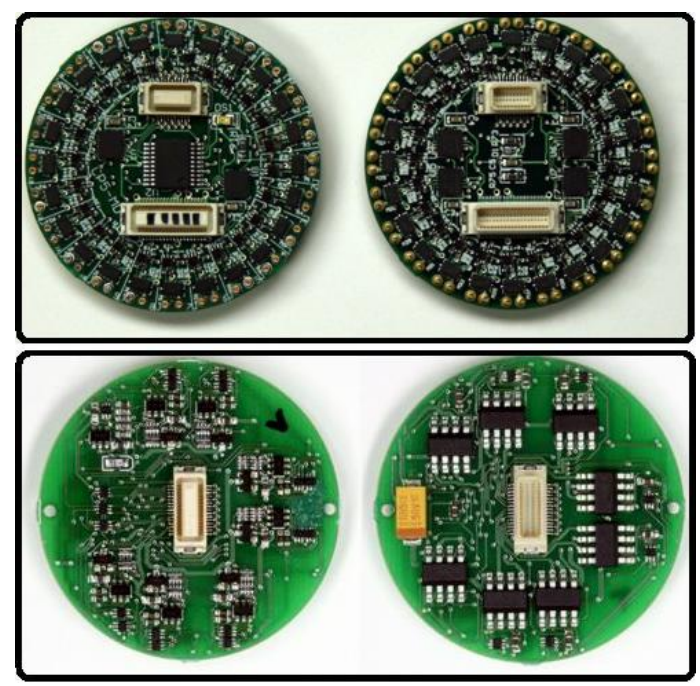

Fig. 6. Density comparison of implementing independent full bridges (top) vs. multiplexed half-bridges (bottom).

high excitation pulse, we can also generate our low power holding currents that can remain on continuously without harming the electromagnets.

Our current electronics are capable of continuously delivering up to $1.5 \mathrm{~A}$ at up to $50 \mathrm{~V}$. Higher voltages exceed the rating of our high density interconnect and approach the breakdown voltage of our existing semiconductors. Given that this power level is sufficient to cause thermal breakdown in our coils in a matter of seconds, our duty cycles are limited solely by the electromagnets and not our drive electronics.

\section{E. Discussion}

We found that the two most important factors in achieving a robust system are the effective magnet torque and the manufacturing precision. Despite several iterations focused solely on maximizing the torque generated, we have only been able to generate four times the torque needed under ideal conditions. This is barely adequate to provide for robust locomotion, as even small misalignments of the magnets can disrupt the system dramatically due to the non-linear reduction of magnetic force. Angular misalignments of the magnets orthogonal to the plane of motion are especially severe as it imparts torques that actually impede motion. Thus, repeatable and precise manufacturing was critical to creating robust designs and required several iterations.

\section{POWER}

Keeping each modular robot in the ensemble fully powered is one of the main challenges in building large scale ensembles. This is particularly true as the modules shrink in size because energy output of batteries does not scale well. In keeping with the ensemble axiom, we take as one of our design constraints that the individual units should not require long term power storage, nor should they require an initial charge when they begin operation. Clearly, providing each robot with a tether to a power source is untenable. The ensemble axiom instructs us to minimize self-supporting

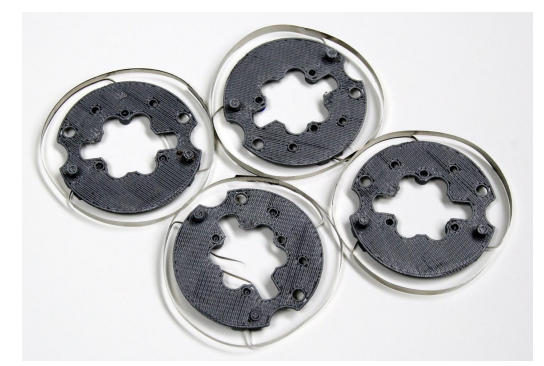

Fig. 7. Using mechanical contacts for power conduction.

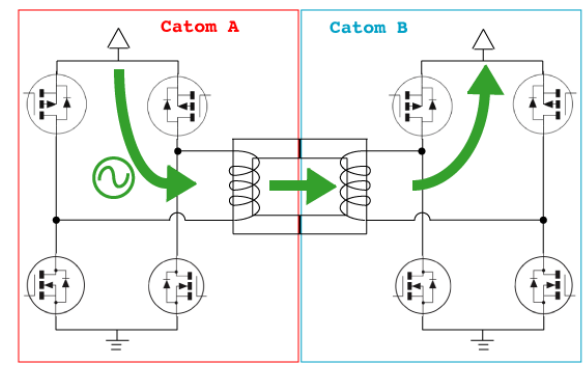

Fig. 8. An $A C$ wave generated in Catom $A$ induces a current in the coupled magnet of Catom $B$. This current is passively rectified via the $h$ bridge protection diodes, providing $B$ with power.

robots and instead provide mechanisms for the units in the ensemble to share and distribute power. We envision a system in which a few of the units are attached to power sources and then through cooperation distribute power amongst the mass.

Our initial attempts at sharing power relied on a power floor and conductive feet as in the Fracta [9] system and the NanoWalker [7]. While effective, it led to unpredictable angular misalignment, preventing robust motion. We next tried DC connections between the units (Figure 7). Such a system requires balancing the need for low resistance electrical contacts between units and the need to keep the spring force and friction between the contacts as low as possible so the power rings don't impede stable movement. While this system worked, it was very sensitive to variations in assembly and neighbor orientation, making it difficult to implement in large numbers. Additionally, it does not scale well to smaller modules as the resistivity will limit the reach. It also requires additional modules to be placed on each robot, reducing its scalability into 3D systems.

One way to eliminate additional modules is to capitalize on the connectivity of the large, high power electromagnets between catoms and transfer power inductively. When two catoms are adjacent the flux shunts of their magnets touch and they form a crude but effective transformer. The electromagnet control circuitry is flexible enough to generate $\mathrm{AC}$ waveforms, allowing one catom to induce currents in the other as shown in Figure 8. Interestingly, the protection diodes in the h-bridges (in this case provided by the body diode behavior of MOSFETs) act as a full bridge rectifier, meaning that power generation on the receiving catom is completely passive and allows disabled catoms to be powered 


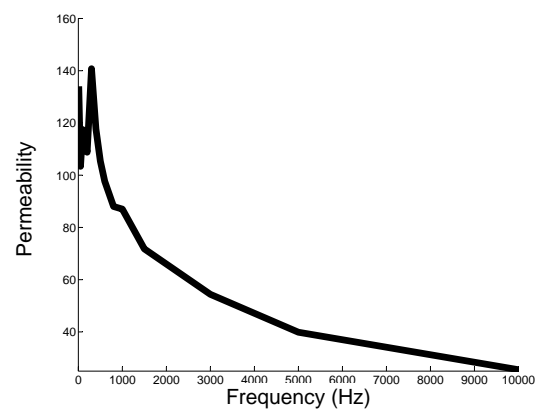

Fig. 9. The effective permeability of the ferrite core decreases as frequency increases.

on. Eliminating the need for direct electrical contact between modules expands the construction options at other scales where low resistance contacts are not possible.

Through simple experimentation we can achieve $0.3 \mathrm{~J} / \mathrm{s}$ of continuous power transfer using a simple square wave at $3 \mathrm{kHz}$ at 15 percent efficiency. As movement requires anywhere from 3-10J, significant energy storage is required. In testing we used an aerogel capacitor array, tied to the system power bus, to provide $.4 \mathrm{~F}$ at $30 \mathrm{~V}$. This fit on the catoms and provided the necessary working voltage. The extremely large energy storage, $180 \mathrm{~J}$, was needed to prevent significant voltage decay during a motion discharge. A more complicated system, where the charge storage is isolated from the main system power, would allow us to adjust the voltage dynamically through standard dc-dc conversion and reduce the needed energy storage. Regulation also compensates for the voltage losses inherent in rectification and transformer coupling, which otherwise limit power transfer to a few hops.

There are many issues that must be addressed before inductive power can efficiently transfer sufficient power to become practical. Clearly, higher rates of power transfer must be achieved if the catoms are to charge, move, and recharge in a reasonable time. A main issue is electromagnet construction: the coils are wound on the core and then two shunts are loose-fit onto the ends, creating a square horseshoe. These loose-fit connections introduce slight air gaps, but they could be ameliorated with a press-fit connection. An alternative would be to wind the coil onto a half-torus, which while more expensive to produce would have a minimum flux path, further increasing efficiency.

The fairly high coil resistance of $28 \Omega$ also creates constraints. This was originally done to minimize the total current needs of the electromagnet. Each catom module has many layers of connectors between the power source, $h$ bridges, and electromagnets, and by reducing the current we reduce the power lost in this interconnect resistance. Also, since our design goal was to develop as high a torque as possible, we use many turns to saturate the core and achieve near-maximum flux density. Together these decisions suggest we use frequencies in the $\mathrm{kHz}$ for power transfer.

Unfortunately, explorations into $\mathrm{kHz}$ frequency waveforms

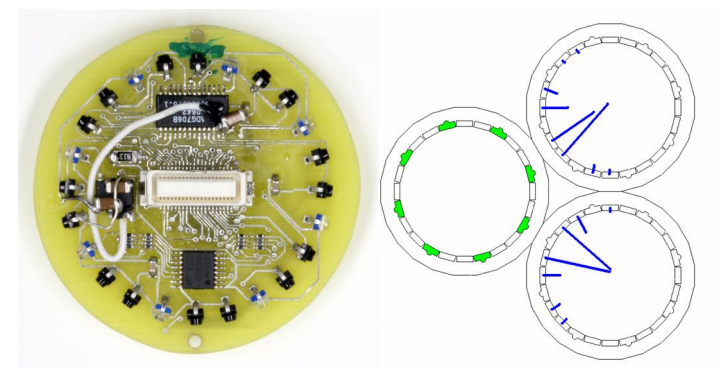

Fig. 10. (a) IR communication and localization modules. (b) Simple vector summation localizes two catoms.

reveal additional problems with the current electromagnet construction. We chose a ferrite core, as low carbon steel is inexpensive to machine and has a high maximum flux density, which is critical to generating high torques. It also has a fairly low starting permeability, which means a significant amount of energy is lost establishing the initial magnetic field. This is not a problem when using DC for torque generation, but at high frequencies most of the energy is lost constantly establishing, destroying, and re-establishing the field [8]. Figure 9 shows the results of analysis that confirms the poor high frequency behavior of the current core. This can be best avoided by using a different core material, one with characteristics better matching the new requirements. Pure iron or an exotic like permandur are suitable as a magnetic material, but their other material properties, as well as cost, make them impractical. Silicon steel is a reasonable compromise: it handles higher frequencies dramatically better than carbon steel, while its slightly lower flux density only minimally reduces the developed torque.

\section{Communication And SEnsing}

Communication is perhaps the most important part of a catom module. Ensemble actions such as movement require coordination between catoms, and global communication between catoms and the outside world greatly simplifies maintenance tasks such as reprogramming, debugging, and program interfacing. While it may be tempting to use only a global broadcasting method, local neighbor-neighbor communication is required for two reasons. First, it has been shown to scale in the control of large claytronic systems [1]. Second, it is capable of providing the minimal sense feedback we need to allow for accurate motion coordination without additional components.

Our initial investigations into local communications used infrared (IR) emitter and detection systems. By having many transmitters and receivers, a catom communicates independently with all of its neighbors. Our most recent system uses 8 transmitters and 16 receivers staggered equally around the perimeter of the catom as in Figure 10a. Increasing the number of receivers gives more angle information, enabling catoms to localize to their neighbors and determine which faces are connected.

Localization via IR is a straightforward process. One catom broadcasts a high value pulse on all of its transmitters 
while its neighbors simultaneously check their sensor values. By doing a vector sum of the resulting data as in Figure 10b, each neighbor can determine which face is most likely in contact with the transmitting catom. This simple sensing is all that is needed to allow coordinated relative motion, as well as provide for closed loop control.

A system currently under investigation uses the electromagnets in a similar manner to the inductive power transfer in Section IV. By transmitting power in a coherent sequence, the receiving module can decode the sequence into a data stream. Since this transmission can only occur between tightly coupled faces, it provides very localized communications with far less likelihood of spurious or reflected signals than an IR system. It also has the significant advantage of required only our existing set of unary actuators.

Unlike our power transfer method which utilizes the existing electronics, inferring the source of inductive data transfer requires additional sensing hardware. One implementation places a small sense resistor on the unshared end of each of the 24 coils. A mirrored current monitor would provide a voltage corresponding to the instantaneous current through the coil, which could be sampled by the microcontroller. Externally induced currents can easily be inferred by the lack of an existing control signal presently driving that coil. Useful communication speeds would require extremely high sampling rates, but localization would require only the grossest sensing capabilities.

While local communication systems are key to research investigation, global communication is a practical necessity for maintaining modules in a laboratory setting. For global communications we use a packet-based API over a 802.15.4 network using Maxstream Xbee modules. This enables serial-speed communications between individually addressable catoms or a host computer, as well as a general broadcast mode. This is used for module firmware upgrades, starting and stopping running programs, as well as interactively querying and setting module state.

\section{CONCLUSIONS}

The planar catoms are a successful application of the claytronics design principles. While catoms at a $45 \mathrm{~mm}$ scale are implemented very differently than at the submillimeter scale, our results in locomotion, power transfer, communication, and programming environments seem promising in their applicability. Of particular value is the idea that a simple pattern of unary contact features enables a single module to participate in and contribute to the ensemble.
By using electromagnets as actuators we demonstrate moving robots without moving parts or mechanisms. Careful calculation and assembly accuracy is needed in the construction of the magnet array, but these issues are commonly addressed in miniature manufacturing and do not rule out scaling down in size. While originally intended only for locomotion and adhesion, the inductive coupling offered by the magnet coils has also proven useful for power transfer, communication, and sensing. This ability to use a single effector in multiple roles further reduces the mechanical complexity of a catom.

Multi-purpose magnetic force effectors are a first step towards scalable claytronic hardware. Distilling the complexity of a robotic module into an array of identical features greatly reduces the domain of design constraints that must be addressed during miniaturization.

\section{REFERENCES}

[1] Burak Aksak, Seth Copen Goldstein, et al. Demo abstract: Claytronics-highly scalable communications, sensing, and actuation networks. In Proceedings of the 3rd international conference on Embedded networked sensor systems (SenSys), page 299, 2005.

[2] J. Bishop, S. Burden, E. Klavins, R. Kreisberg, W. Malone, N. Napp, and T. Nguyen. Self-organizing programmable parts. In International Conference on Intelligent Robots and Systems. IEEE/RSJ Robotics and Automation Society, 2005.

[3] Reston Condit and Douglas W. Jones. Stepping motors fundamentals. http://ww1.microchip.com/downloads/en/appnotes/00907a.pdf, 2004.

[4] Seth Copen Goldstein, Jason Campbell, and Todd C. Mowry. Programmable matter. IEEE Computer, 38(6):99-101, June 2005.

[5] M.W. Jorgensen, E.H. Ostergaard, and H.H. Lund. Modular atron: modules for a self-reconfigurable robot. In IEEE/RSJ International Conference on Intelligent Robots and Systems (IROS), volume 2, pages 2068-73, September 2004.

[6] Brian Kirby, Jason Campbell, Burak Aksak, Padmanabhan Pillai, James F. Hoburg, Todd C. Mowry, and Seth Copen Goldstein. Catoms: Moving robots without moving parts. In AAAI (Robot Exhibition), pages 1730-1, Pittsburgh, PA, July 2005.

[7] S. M. Martel, T. Koker, S. Riebel, M. Sherwood, J. Suurkivi, and I. W. Hunter. Infrastructure suited for supporting a fleet of wireless miniature robots designed for atomic-scale operations. In Proc. SPIE, Microrobotics and Microassembly III, volume 4568, pages 221-230, October 2001.

[8] Colonel Wm. T. McLyman. Transformer and Inductor Design Handbook. Marcel Dekker, Inc., 1988.

[9] S. Murata, H. Kurokawa, and S. Kokaji. Self-assembling machine. In Proc. IEEE Int. Conf. Robotics and Automation, pages 441-8, 1994.

[10] Satoshi Murata, Eiichi Yoshida, Akiya Kamimura, Haruhisa Kurokawa, Kohji Tomita, and Shigeru Kokaji. M-tran: Selfreconfigurable modular robotic system. IEEE/ASME Trans. on Mechatronics, 7(4), Dec 2002.

[11] White P., Zykov V., Bongard J., and Lipson H. Three dimensional stochastic reconfiguration of modular robots. In Proceedings of Robotics Science and Systems, Cambridge, MA, June 2005.

[12] J.W. Suh, S.B. Homans, and M. Yim. Telecubes: mechanical design of a module for self-reconfigurable robotics. In Proc. of the IEEE Int'l Conference on Robotics and Automation (ICRA), volume 4, pages 4095-101, 2002. 